\title{
Integrated electroplated heat spreaders for high power semiconductor lasers
}

\author{
Jianping Fu, ${ }^{1, a)}$ Ronggui Yang, ${ }^{1, b)}$ Gang Chen, ${ }^{1, c)}$ Jean Pierre Fleurial, ${ }^{2}$ and \\ G. Jeffrey Snyder ${ }^{2}$ \\ ${ }^{1}$ Department of Mechanical Engineering, Massachusetts Institute of Technology, 77 Massachusetts Avenue, \\ Cambridge, Massachusetts 02139, USA \\ ${ }^{2}$ Jet Propulsion Laboratory, 4800 Oak Grove Drive, Pasadena, California 91109, USA
}

(Received 9 June 2008; accepted 3 August 2008; published online 22 September 2008)

\begin{abstract}
Thermal management of high power semiconductor lasers is challenging due to the low thermal conductivity of the laser substrate and the active device layers. In this work, we demonstrate the use of a microfabricated laser test device to study the thermal management of edge emitting semiconductor lasers. In this device, metallic heat spreaders of high thermal conductivity are directly electroplated on structures that mimic edge-emitting semiconductor lasers. The effects of various structural parameters of the heat spreader on the reduction of the thermal resistance of the laser test device are demonstrated both experimentally and theoretically. Without resolving to computational costive simulations, we developed two independent analytical models to verify the experimental data and further utilized them to identify the dominant thermal resistance under different laser mounting configurations. We believe our approach here of using microfabricated devices to mimic thermal characteristics of lasers as well as the developed analytical models for calculating the laser thermal resistance under different mounting configurations can potentially become valuable tools for thermal management of high power semiconductor lasers. () 2008 American Institute of Physics. [DOI: 10.1063/1.2986888]
\end{abstract}

\section{INTRODUCTION}

High power semiconductor lasers have found broad applications in solid-state laser pumping, direct material processing, medical surgery, printing, and manufacturing. ${ }^{1,2}$ However, the maximum optical output power of semiconductor lasers is limited by the temperature rise in the laser active region (e.g., the so-called thermal rollover effect). ${ }^{3-5}$ Depending on the thermal resistance of the semiconductor laser, the heat generation in the laser active region results in a temperature rise that reduces the carrier confinement and increases the nonradiative recombination processes. ${ }^{2,6}$ Both effects lead to a higher threshold current and a lower differential efficiency, leading to a limited maximum output power of the laser. The self-heating of the lasers also causes other effects that degrade laser performance. For example, the emission wavelength of a semiconductor laser shifts with temperature, since the refractive index of the lasing region as well as the length of the laser cavity increase with temperature. When operating a semiconductor laser at a high output power, the laser device also often shows beam distortion in the lateral direction, which implies that there are hot regions inside the cavity where the refractive index increases leading to parasitic optical waveguides, which destroy the lateralmode profile. ${ }^{6}$

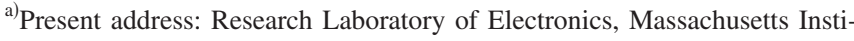
tute of Technology, 77 Massachusetts Avenue, Cambridge, MA 02139, USA.

${ }^{b}$ Present address: Department of Mechanical Engineering, University of Colorado at Boulder, Boulder, CO 80309, USA.

c) Author to whom correspondence should be addressed. Tel.: + 1 (617) 2530006. FAX: +1 (617) 258-5802. Electronic mail: gchen2@mit.edu.
}

The thermal characteristics and thermal management of semiconductor lasers have been investigated by various researchers. ${ }^{7-14}$ Most of these thermal management research works use the junction-side-down mounting configuration to spread the heat out, either passively through high thermal conductivity substrates ${ }^{7-11,13}$ or actively through integrated microfluidic cooling systems. ${ }^{12,14}$ The laser is bonded to heat spreader through relatively low thermal conductivity solder materials, which can limit the laser performance. ${ }^{7,13}$ More recently, the use of metallic heat spreaders directly attached on top of the laser by either metal electroplating, sputtering, or low-temperature metal-to-chip direct bonding has been proposed to reduce the thermal resistance between the laser active heating region and the external cooling system. ${ }^{2}$ With its high thermal conductivity, a directly attached metallic heat spreader eliminates the use of the low thermal conductivity interface materials between the laser bar and the heat spreader, thus avoiding the bonding process and the interface imperfections often generated in the soldering process. For example, voids in the soldering layer have been found to give rise to hot spots and therefore increase the thermal resistance. ${ }^{8}$ In this work, to investigate the effectiveness of directly plated metallic heat spreaders, we propose to use a microfabricated laser test structure to model thermal characteristics of high power semiconductor lasers. The microfabricated laser test structure was designed and fabricated with integrated metallic heaters and temperature sensors, and copper heat spreaders of different dimensions (e.g., width and height) were directly plated on top of the laser test structure. The thermal resistances of the laser test samples were measured with junction-side-up mounting configuration, which has been reported to be more effectively improved by the 


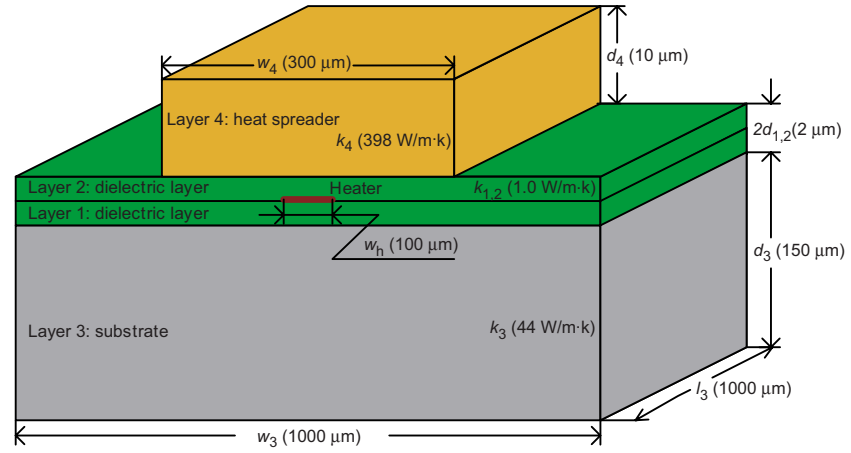

FIG. 1. (Color online) Schematic illustration of the heat spreader test device. The metallic heater stripe is embedded between two dielectric layers. A thick metallic layer of high thermal conductivity is electroplated on top of the test structure to serve as a heat spreader. Nominal thermal conductivities and dimensions of the different structural layers are indicated in the parenthesis (not to scale; heat spreader: copper, dielectric layers: silicon dioxide or silicon nitride, substrate: GaAs).

heat spreader structure. ${ }^{13}$ Two independent analytical models were developed to verify the thermal resistance experimental data and further were utilized to identify the dominant thermal resistance of the heat spreader devices under different mounting configurations, thus providing engineering design guidelines of the hear spreader structures for improving the thermal management of high power semiconductor lasers.

\section{DEVICE DESIGN AND FABRICATION}

We aim at mimicking edge emitting laser structures, ${ }^{15}$ including high-power quantum-well edge-emitting lasers and quantum cascade lasers. ${ }^{16}$ In the quantum-well based edgeemitting lasers, cladding layers are used to confine the carriers and the light. These cladding layers are typically made of alloys that have low thermal conductivity. Heat is generated in both the cladding layers by resistive heating and the active region by the nonradiative recombination. In quantum cascade lasers, thick superlattice structures are used as the lasing region, which experiences both resistive heating and nonradiative recombination heating. The superlattice structures have even lower thermal conductivity than their equivalent alloys, leading to more severe heating problems in quantum cascade lasers. ${ }^{17}$ Here we designed and fabricated electroplated copper heat spreader test structures to model the thermal characteristics of semiconductor lasers (Fig. 1). In the design, a heater stripe (of width $w_{h}$ ) was embedded between two dielectric layers of low thermal conductivity. By controlling the thickness of the dielectric layers $\left(d_{2,3}\right)$, their thermal resistances can be matched comparably to those of the actual laser device layers. The metallic heater also served as a resistive thermometer to determine the temperature rise under different heating power conditions. Different sets of heat spreaders with different widths $\left(w_{4}\right)$ and heights $\left(d_{4}\right)$ were fabricated to investigate the effect of the heat spreader geometrical parameters on the thermal resistance.

The electroplated copper heat spreader structure was fabricated with conventional semiconductor microfabrication techniques in the clean room environment (Fig. 2). A 2 in. GaAs wafer was chosen as the substrate because many semiconductor lasers are manufactured with this material. Other

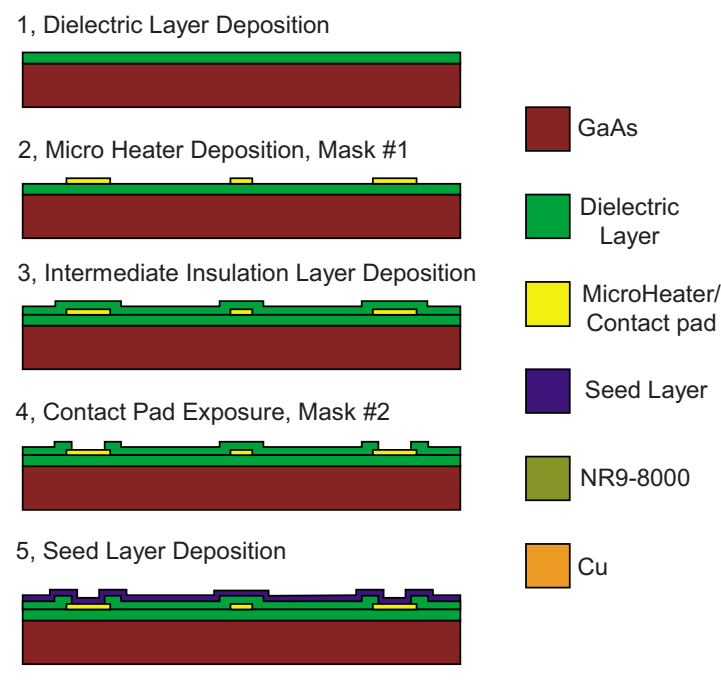

6, NR9-8000 Photoresist Mold Pattern, Mask \#3

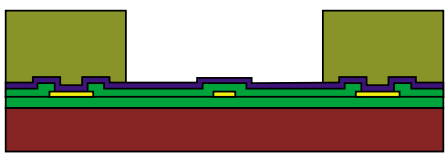

7, Cu Electroplating

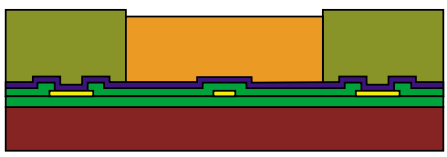

8, Photoresist Mold Removal

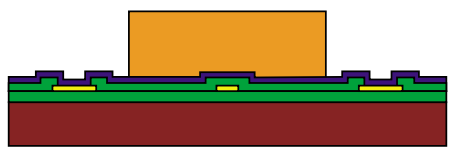

9, Seed Layer Removal

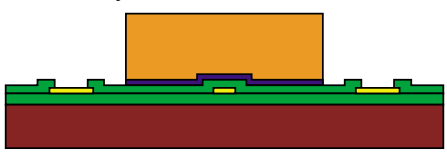

FIG. 2. (Color online) Fabrication process flow of the integrated copper heat spreader device.

substrate materials for semiconductor lasers, such as GaSb, have similar thermal properties with GaAs. In our experiments, test structures with $\mathrm{Si}$ wafer as a substrate were also fabricated with the same fabrication process for device performance comparison. A $1 \mu \mathrm{m}$ thick dielectric layer (silicon nitride or silicon dioxide) was first deposited on the substrate by the plasma enhanced chemical vapor deposition (PECVD) method in multiple steps. The multideposition of dielectric layers was needed to reduce the number of pinholes and thus ensure the metallic heater pattern would be completely electrically insulated from the substrate. ${ }^{18}$ Since our experimental measurements made use of the linear temperatureresistance relation of the metallic heater, any current leakage between the heater pattern and the substrate would ruin the measurement. After the first dielectric layer deposition, the first photolithography step was performed to define the metallic heaters. A multilayer of $\mathrm{Ti} / \mathrm{Pt} / \mathrm{Au} / \mathrm{Ti}$ $(200 \AA / 300 \AA / 1500 \AA / 200 \AA)$ was deposited by electron-beam evaporation and followed by lift-off to form 
the heaters. The two titanium layers were adhesion layers and the platinum layer served as a diffusion barrier for gold. After the lift-off process, another PECVD dielectric layer of about $1 \mu \mathrm{m}$ thick was deposited as the top insulation layer. The electrical contact pads were defined by etching through the second dielectric layer by reactive ion etching method.

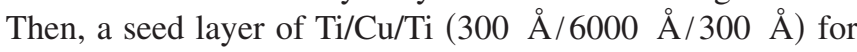
copper electroplating was deposited by electron-beam evaporation. The copper seed layer was of sufficient thickness to ensure uniform plating across the entire wafer with minimal resistivity effect. Both the titanium layers were adhesion layers. It is worth mentioning that the top layer of titanium also served to prevent oxidation of the copper seed film during subsequent processing steps leading up to copper electroplating.

After the seed layer deposition, a negative thick photoresist NR9-8000 (Futurrex, Inc., Franklin, NJ) was spin coated to obtain thick electroplating molds. Photoresist molds thicker than $100 \mu \mathrm{m}$ were obtained with both singlespin and double-spin methods. ${ }^{19}$ After development with the Futurrex RD6 developer (Futurrex, Inc., Franklin, NJ), the thick NR9-8000 mold could be optionally thermally cured to achieve increased resistance to chemical attack during subsequent processing steps. However, this thermal cure often resulted in undesired distortion and shrinkage of the mold pattern and further decreased the height of the photoresist film as well as compromised the sharpness of the mold sidewalls. Therefore, the heat spreader structures described in this work used NR9-8000 molds that were not thermally cured prior to electroplating. The topmost titanium layer was removed by a quick dipping into diluted buffered-oxide etchant (BOE) to expose the thick copper seed layer. After a de-ionized (DI) water rinse of the wafer, a short ultrasonic treatment of the wafer in DI water was carried out to remove the entrapped air bubbles to ensure intimate contact between the plating solution and the seed layer during plating.

Copper electroplating was performed using a commercially available acid copper plating solution (Technic Copper RTU-type, Technic, Inc., Anaheim, CA). The size of the copper anode was designed to be about $5 \times 5 \mathrm{~cm}^{2}$, so that the ratio of the anode to the cathode area (i.e., the wafer) was about 1:1. At the beginning of the electroplating, the dc current was set to a low value and increased stepwise slowly to ensure uniform copper plating. The slow current ramping ensured that the wetting agent in the plating bath adhered to the top of cathode to provide good surface wetting, which aided in bubble prevention. Stresses in the plated copper were also minimized by starting with a small plating current. ${ }^{20}$ For all the experiments, the plating current density was limited to $20 \mathrm{~mA} / \mathrm{cm}^{2}$, leading to an approximate copper plating rate of $0.4-0.5 \mu \mathrm{m} / \mathrm{min}$. It took about $3 \mathrm{~h}$ to plate an $80 \mu \mathrm{m}$ thick copper in the photoresist mold. After electroplating was complete, the NR9-8000 photoresist mold was completely dissolved with an acetone bath.

Electrical isolation of the electroplated heat spreaders was achieved by etching away the seed layers. A brief dip in dilute BOE was performed to remove the two titanium layers separately. A dilute nitric acid bath together with citric acid $\left(\mathrm{C}_{6} \mathrm{H}_{8} \mathrm{O}_{7} \mid \mathrm{H}_{2} \mathrm{O}\right)$ was used to remove the copper seed layer.
The citric acid is believed to reduce the etch rate of copper in nitric acid and to reduce the nonuniformity of the finished copper surface. ${ }^{21}$ Finally, the wafer was cut into individual devices for further testing.

\section{EXPERIMENTAL DATA}

Experiments were performed with two different sets of test devices, one with electroplated copper heat spreaders (as shown in Fig. 2, step 9, referred as "heat spreader device") and the other without, but all the other structural layers were identical (as shown in Fig. 2, step 4, referred as "reference device"). The latter served to gauge the effectiveness of the electroplated copper heat spreader for reducing thermal resistance of the heat spreader device. The experimental procedure was divided into two sequential steps. The first step was to characterize the temperature coefficient of resistance (TCR) of each device under a low current loading ( $\leq 1 \mathrm{~mA}$ ), and then the following second step was carried out to measure thermal resistances of the devices under the junction-size-up mounting configurations.

Before experiments, all the test devices were mounted on a copper heat sink ( 4 in. diameter, 1 in. thickness) in the junction-side-up fashion with a thin thermal grease layer. A fine type- $K$ thermocouple (Omega Engineering, Inc., Stamford, $\mathrm{CO}$ ) was attached in close proximity to the device on top of the copper sink. This type- $K$ thermocouple was used to determine the metallic heater temperature during the TCR calibration and also to measure the temperature at the device bottom surface during the thermal resistance measurement. The TCR of all the devices were calibrated by measuring the electrical resistances of the metallic heaters under a low electrical current loading using the four-probe method while they were heated in an isothermal environment to different temperatures [Fig. 3(a)]. It is worth mentioning that the TCR measurements were performed only after the devices had passed through one initial thermal cycle to ensure that reproducible results could be obtained in subsequent measurements. This thermal treatment is believed necessary due to the annealing process of the metallic layers encountered during the initial thermal cycle.

The thermal resistance measurement was carried out by passing electrical currents of different magnitudes through the metallic heater. The metallic heater was heated electrically and its resistance increased with the temperature. The electrical resistance and the heating power were determined by measuring the electrical current and the voltage drop across the heater from the four leads. Knowing the TCR from the first measurement step, the heater temperature was calculated from its resistance. The temperature of the device bottom surface was monitored at the same time by the type- $K$ thermocouple. Figure 3(b) shows a typical set of experimental data of the temperature difference $\Delta T$ between the metallic heater and the device bottom surface as a function of the heating power $P$. The thermal resistances $R$ of the devices were determined by linearly fitting the data to obtain the slopes of the curves.

Figures 3(c) and 3(d) show the dependence of thermal resistance $R$ of GaAs- and Si-based heat spreader devices on 


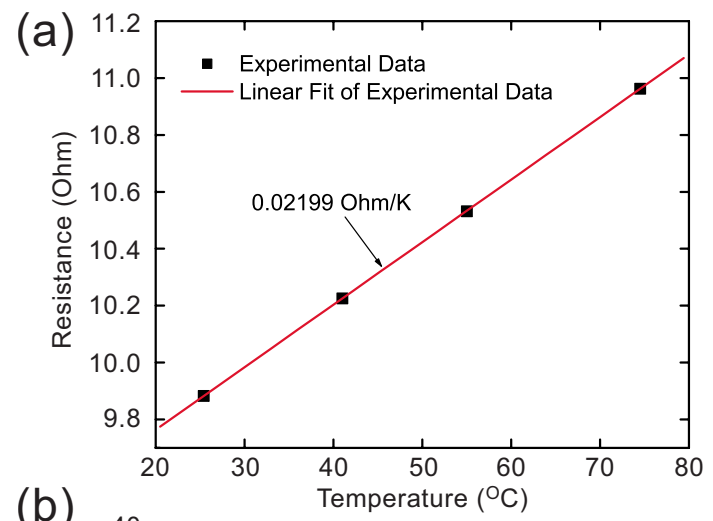

(b)

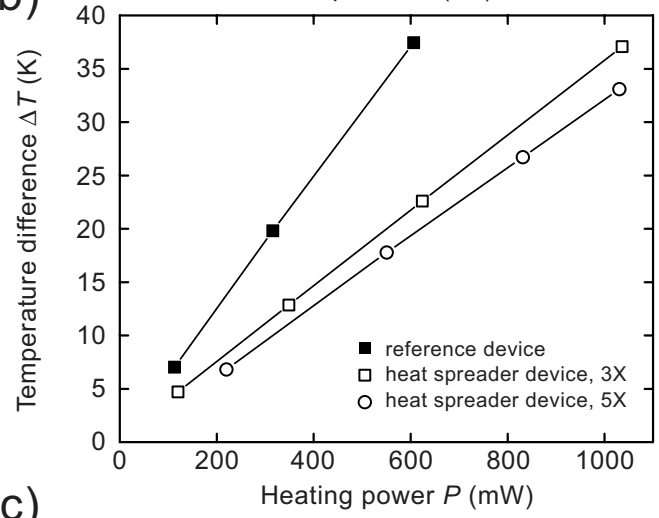

(c)

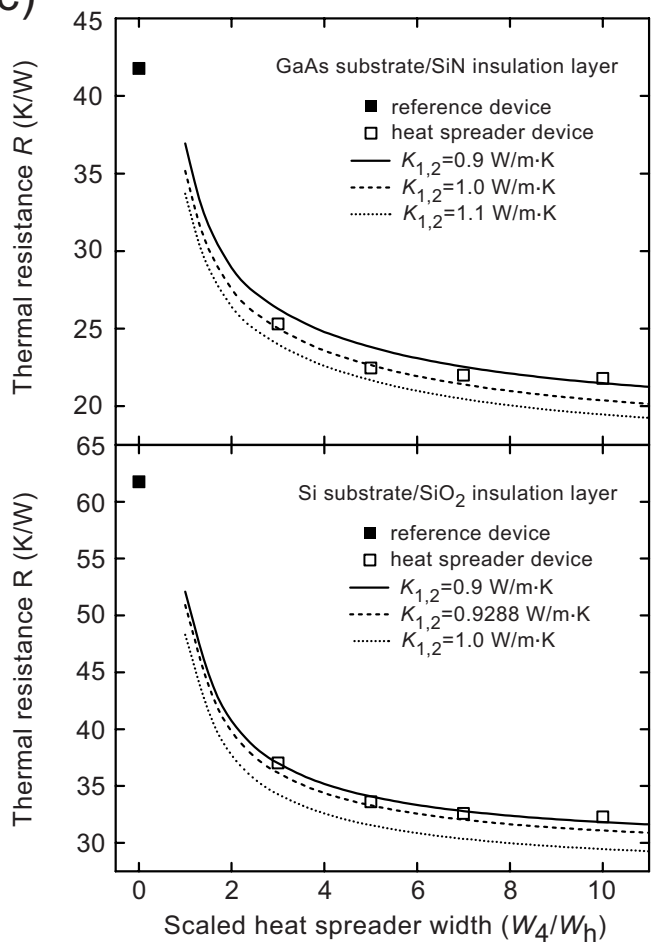

FIG. 3. (Color online) (a) Four-probe electrical resistance of the metallic heater in a GaAs sample as a function of temperature. (b) Temperature difference $\Delta T$ between the metallic heater and the device bottom surface as a function of heating power $P$. Experimental data are from devices with $\mathrm{Si}$ as substrate and $\mathrm{SiO}_{2}$ as dielectric layers. Heater dimension: $w_{h}=360 \mu \mathrm{m}$, $l_{3}=100 \mu \mathrm{m}$. Copper heat spreader thickness $d_{4}=80 \mu \mathrm{m}$. The numerical numbers indicated inside the figure are the scaling factor of $w_{4} / w_{h}$. [(c) and (d) ] Thermal resistance $R$ of (c) GaAs-based and (d) Si-based devices as a function of the scaling factor of $w_{4} / w_{h}$. GaAs devices: $w_{h}=750 \mu \mathrm{m}, l_{3}$ $=120 \mu \mathrm{m}$, and $d_{4}=80 \mu \mathrm{m}$. Si devices: $w_{h}=360 \mu \mathrm{m}, w_{3}=100 \mu \mathrm{m}$, and $d_{4}=80 \mu \mathrm{m}$. Reference samples are included for comparison. Different thermal conductivity values of the dielectric layers are used for fitting as indicated. In particular, $k_{1,2}=0.9288 \mathrm{~W} / \mathrm{m} \mathrm{K}$ was determined by fitting the thermal resistance of the Si reference device (d). the heat spreader width $w_{4}$, respectively. The dielectric layers deposited on Si devices were PECVD silicon dioxide, which was different from the silicon nitride layer deposited on the GaAs devices. The PECVD silicon dioxide layer was found to serve better as the insulation layer to prevent current leaking. As seen clearly from both Figs. 3(c) and 3(d), the thermal resistances of the heat spreader devices initially decreased quickly with the heat spreader width $w_{4}$ and then leveled off with a lower bound value of about half the thermal resistance value of the reference devices. Most of the heat generated in the metallic heater region flows up to the top plated copper layer, spreads out, and then flows downwards through the test structure over a large area, resulting in reduction of the thermal resistance $R$. Most of the benefit comes from the first few scaled heat spreader width (defined here as $\left.w_{4} / w_{h}\right)$. With a scaled heat spreader width of 5 , the thermal resistances of both GaAs- and Si-based heat spreader devices already reduce about $50 \%$ with the junction-side-up mounting configuration.

\section{ANALYTICAL MODELING}

In principle, the thermal resistance $R$ of the heat spreader test structure can be calculated from numerical solutions (e.g., the finite difference and the finite element methods). However, the great dimensional disparities existing in the test structures (i.e., $1 \mu \mathrm{m}$ thick dielectric layers compared with millimeter-sized substrate) as well as the irregular meander-shaped heater pattern make these numerical methods time consuming and ineffective. Moreover, both the finite difference and the finite element methods provide solutions only for individual problems. Therefore, it is relatively difficult from these numerical methods to deduce general guidelines for thermal management of high power semiconductor lasers. In this work, we have developed two different analytical models to calculate the thermal resistance $R$ of the heat spreader device based on the separation of variables solution of Laplace's equation for two-dimensional rectangular substrate plate. In both of the analytical models, heat $(Q)$ is assumed to be generated uniformly and steadily in the planar heater. At a heat source free interface between any two layers, the perfect thermal contact boundary conditions are assumed with continuous temperature and normal heat flow. No heat escapes through the sides or ends of the heat spreader structure, and all the other surfaces exposed to the ambient are treated as adiabatic since the natural convection and radiation loss is estimated to be negligible. For different laser mounting configurations, the interface between the laser device and the heat sink is assumed to be maintained at the same temperature as the heat sink $\left(T_{0}\right)$. In both of the analytical models, we have neglected the contribution of the thin seed layers for the thermal resistance $R$ of the heat spreader device [Fig. 4(a)].

\section{A. First simplified analytical model}

This analytical model is constructed by representing the heat spreader device as a thermal circuit of individual thermal resistances [Fig. 4]. Expressions of all the thermal resistance components are derived first, and then the total thermal 
(a)
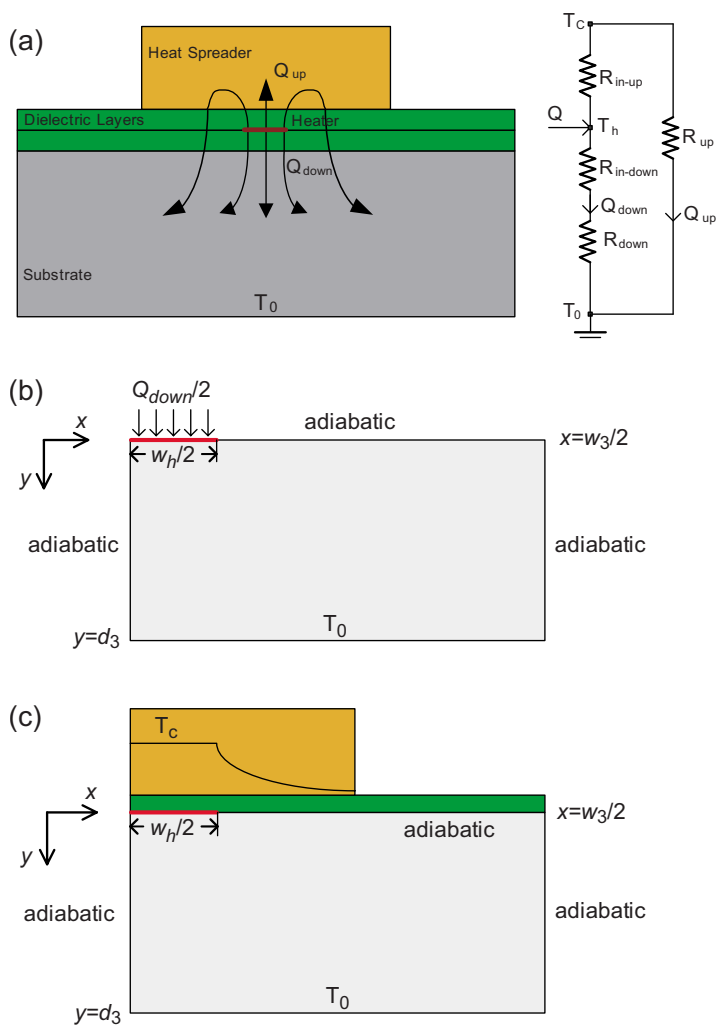

FIG. 4. (Color online) (a) Schematics of heat flow in the heat spreader test device for the junction-side-up mounting configuration. The corresponding thermal circuit is plotted on the right. [(b) and (c)] Boundary conditions of the substrate plate for calculating the thermal resistance of (b) $R_{\text {down }}$ and (c) $R_{\text {up }}$, respectively. Only the right half part of the substrate is considered due to the symmetry. Positive $y$ direction points away from the heater region with its origin at the center of the substrate top surface.

resistance $R$ of the heat spreader device is summed up accordingly. In the model, heat $Q$ generated in the meandershaped heater is divided into two parts. The first part of the heat $Q_{\text {down }}$ flows down directly through both the bottom dielectric layer and the substrate into the heat sink, and the rest $\left(Q_{\text {up }}=Q-Q_{\text {down }}\right)$ enters the heat spreader through the top dielectric layer and spread laterally. $Q_{\text {up }}$ re-enters the two dielectric layers and the substrate, and finally flows into the heat sink. For $Q_{\text {down }}$, heat flow in the bottom dielectric layer can be safely assumed to be one-dimensional, and thus the corresponding thermal resistance $R_{\text {in-down }}$ is calculated as $R_{\text {in-down }}=d_{1} /\left(k_{1} \cdot A_{\text {heating }}\right)$ (here $A_{\text {heating }}$ represents the actual heating area of the irregular meander-shaped heater). The two-dimensional heat flow of $Q_{\text {down }}$ in the substrate has thermal resistance of $R_{\text {down }}$. For $Q_{\mathrm{up}}$, heat flow in the top dielectric layer is assumed to be one-dimensional and the corresponding thermal resistance $R_{\text {in-up }}$ is equal to $R_{\text {in-up }}$ $=d_{2} /\left(k_{2} \cdot A_{\text {heating }}\right)$. Thermal resistance from the top heat spreader layer to the substrate bottom is calculated as $R_{\text {up }}$, and here the effects of the two dielectric layers and the heat spreading of the heat spreader are included into the boundary conditions of the top side of the substrate when calculating $R_{\text {up. }}$

The thermal resistances $R_{\text {down }}$ and $R_{\text {up }}$ are calculated from the separation of variable solution of Laplace's equation for two-dimensional rectangular substrate plate with left and right sides adiabatic, and bottom side at a constant tem- perature $T_{0}$ (Fig. 4). Therefore, the dimensionless temperature distribution of $\theta_{3}\left[\theta_{3}=\left(T_{3}-T_{0}\right) / T_{0}\right]$ in the substrate (or layer 3) can be expressed as ${ }^{22}$

$$
\begin{aligned}
\theta_{3}(x, y)= & \beta_{3,0}\left(1-\gamma_{3,0} y\right)+\sum_{n=1}^{\infty} \beta_{3, n}\left[\cosh \left(\alpha_{n} y\right)\right. \\
& \left.-\gamma_{3, n} \sinh \left(\alpha_{n} y\right)\right] \cos \left(\alpha_{n} x\right),
\end{aligned}
$$

where $\beta_{3, n}$ is the undetermined separation coefficient of $\cosh \left(\alpha_{n} y\right) \cos \left(\alpha_{n} x\right)$ and $\gamma_{3, n}$ is the ratio of the coefficients of the sinh and cosh terms. In Eq. (1), $\sin \left(\alpha_{n} x\right)$ does not appear because of the symmetric stripe position, and the separation constant $\alpha_{n}$ is $2 n \pi / w_{3}$ in order to satisfy the requirement of no heat escape from the lateral faces. The local thermal resistance $R_{\text {down }}(x)$ can be expressed as

$$
R_{\text {down }}(x)=T_{0} \theta_{3}(x, 0) / Q_{\text {down }}=T_{0} \sum_{n=0,1}^{\infty} \beta_{3, n} \cos \left(\alpha_{n} x\right) / Q_{\text {down }} .
$$

The average thermal resistance $R_{\text {down }}$ over the heater stripe area can be calculated as

$$
\begin{aligned}
R_{\text {down }}= & w_{h}^{-1} \int_{-w_{h} / 2}^{w_{h} / 2} R_{\text {down }}(x) d x=\frac{T_{0} \beta_{3,0}}{Q_{\text {down }}} \\
& +\frac{2 T_{0}}{Q_{\text {down }} \cdot w_{h n=1}} \sum_{n=1}^{\infty} \frac{\beta_{3, n}}{\alpha_{n}} \cdot \sin \left(\frac{1}{2} \alpha_{n} w_{h}\right) .
\end{aligned}
$$

The evaluation of $\beta_{3, n}$ is carried out below in a closed form, and therefore determination of $R_{\text {down }}$ reduces to the summation of a sufficient number of terms in Eq. (3). (All of the applications to follow required of the order of 100 terms for three significant figures.)

The evaluation of $\beta_{3, n}$ for $R_{\text {down }}$ is carried out by applying the inhomogeneous boundary conditions at the topside of the substrate to Eq. (1) as [Fig. 4(b)]

$$
\begin{aligned}
& \frac{\partial \theta_{3}(x, 0)}{\partial y}=-\frac{Q_{\text {down }}}{T_{0} k_{3} l_{3} w_{h}}, \quad x \in\left[0, \frac{w_{h}}{2}\right], \\
& \frac{\partial \theta_{3}(x, 0)}{\partial y}=0, \quad x \in\left(\frac{w_{h}}{2}, \frac{w_{3}}{2}\right],
\end{aligned}
$$

Please notice that in Eq. (4) we have assumed that $Q_{\text {down }}$ spreads quickly and becomes uniform across the heating area once it arrives at the top surface of the substrate. By taking advantage of the orthogonal property of $\cos \left(2 n \pi x / w_{3}\right)$, we can multiply both sides of Eq. (4) with $\cos \left(2 m \pi x / w_{3}\right)(m$ $=0,1,2 \ldots)$ and perform integration from $x=0$ to $x=w_{3} / 2$. Therefore, $\beta_{3, n}$ can be directly determined as

$$
\begin{aligned}
& \beta_{3,0}=\frac{d_{3} Q_{\text {down }}}{T_{0} k_{3} l_{3} w_{3}}, \\
& \beta_{3, n}(n>0)=\frac{w_{3} Q_{\text {down }} \tanh \left(\alpha_{n} d_{3}\right) \cdot \sin \left(n \pi w_{h} / w_{3}\right)}{T_{0} k_{3} l_{3} w_{h}(n \pi)^{2}},
\end{aligned}
$$

By combining Eqs. (3) and (5), the thermal resistance $R_{\text {down }}$ can be calculated. 
To calculate $R_{\text {up }}$, we can safely assume the temperature of the heat spreader only varies along the $x$-direction due to the smallness of the Biot number Bi (an equivalent thin "fin" and $\mathrm{Bi} \sim 0.1)$ [Fig. 4(c)]. We further assume the temperature in the heat spreader is uniform above the metallic heater region $\left(T_{c}\right)$, and beside the heater region, the temperature difference between the copper heat spreader and the topside of the substrate decays exponentially along the $x$-direction. The decay constant $\eta$ is estimated from the fin model as $\eta$ $=\left[k_{2} /\left(2 d_{2} d_{3} k_{3}\right)\right]^{1 / 2}$. The dimensionless temperature $\theta_{3}$ in the substrate here is redefined as $\theta_{3}=\left(T-T_{0}\right) /\left(T_{c}-T_{0}\right)$, and therefore $R_{\text {up }}$ can be calculated from Eq. (1) as

$$
\begin{aligned}
R_{\text {up }}= & \frac{T_{c}-T_{0}}{Q_{\text {up }}}=\left[-k_{3} \int_{-w_{3} / 2}^{w_{3} / 2} \frac{\partial \theta_{3}(x, 0)}{\partial y} l_{3} d x\right]^{-1} \\
= & \left\{2 l _ { 3 } k _ { 3 } \left[\frac{\beta_{3,0} w_{4}}{2 d_{3}}+\sum_{n=1}^{\infty} \beta_{3, n} \cdot \operatorname{coth}\left(\alpha_{n} d_{3}\right)\right.\right. \\
& \left.\left.\cdot \sin \left(\frac{n \pi w_{4}}{w_{3}}\right)\right]\right\}^{-1},
\end{aligned}
$$

where $\alpha_{n}$ is $2 n \pi / w_{3}$ in order to satisfy the requirement of no heat escape from the lateral faces. The inhomogeneous boundary conditions at the topside of the substrate can be expressed as

$$
\frac{\partial \theta_{3}(x, 0)}{\partial y}= \begin{cases}\frac{(1-\varepsilon) k_{2}\left[\theta_{3}(x, 0)-1\right]}{2 k_{3} d_{2}}, & x \in\left[0, \frac{w_{h}}{2}\right] \\ \frac{k_{2}\left[\theta_{3}(x, 0)-1\right] \cdot e^{-\eta\left(x-w_{h} / 2\right)}}{2 k_{3} d_{2}}, & x \in\left(\frac{w_{h}}{2}, \frac{w_{4}}{2}\right] \\ 0, & x \in\left(\frac{w_{4}}{2}, \frac{w_{3}}{2}\right] .\end{cases}
$$

Please notice that in Eq. (7) we have assumed an adiabatic boundary condition for $Q_{\text {up }}\left(x \in\left(w_{4} / 2, w_{3} / 2\right]\right)$ on the interface between the substrate and the dielectric layer, since the radiation and convection loss from the substrate is negligibly small and further the heat flow in the dielectric layers is largely one dimensional. To make use of the orthogonal property of $\cos \left(2 n \pi x / w_{3}\right)$, both sides of Eq. (7) are multiplied by $\cos \left(2 m \pi x / w_{3}\right)(m=0,1,2 \ldots)$ and integrated from $x=0$ to $x=w_{3} / 2$. The separation constants $\beta_{3, n}$ cannot be directly calculated as in Eq. (5); however, a set of linear equations with undetermined separation coefficients $\beta_{3, n}$ can be achieved, which has the form of $\beta_{3, n}=\sum^{\infty} M_{n, m} \beta_{3, m}$ (n $=0,1,2 \ldots)$, where $M_{n, m}$ are constants determined solely by the thermal properties and the dimensions of the heat spreader test structure. This linear equation set is solved by computer program, and the solutions of $\beta_{3, n}$ are substituted into Eq. (6) to yield $R_{\text {up }}$.

The thermal resistance $R$ of the heat spreader device and the reference device can be calculated as $R=\left(R_{\text {down }}\right.$ $\left.+R_{\text {in,down }}\right)\left(R_{\text {up }}+R_{\text {in,up }}\right) /\left(R_{\text {down }}+R_{\text {in,down }}+R_{\text {up }}+R_{\text {in,up }}\right)$ and $R$ $=R_{\text {down }}+R_{\text {in,down }}$, respectively, in which $R_{\text {down }}$ and $R_{\text {up }}$ are given by Eqs. (3) and (6), respectively. $Q_{\text {up }}$ and $Q_{\text {down }}$ can
TABLE I. Boundary conditions of the heat spreader top surface and the substrate bottom surface for the junction-side-up, junction-side-down, and sandwich mounting configurations.

\begin{tabular}{lccc}
\hline \hline & Junction-side-up & Junction-side-down & Sandwich \\
\hline $\begin{array}{l}\text { Heat spreader } \\
\text { top surface }\end{array}$ & $\partial \theta_{4}\left(x, t_{4}\right) / \partial y=0$ & $\theta_{4}\left(x, t_{4}\right)=0$ & $\theta_{4}\left(x, t_{4}\right)=0$ \\
$\begin{array}{l}\text { Substrate } \\
\text { bottom surface }\end{array}$ & $\theta_{3}\left(x, t_{3}\right)=0$ & $\partial \theta_{3}\left(x, t_{3}\right) / \partial y=0$ & $\theta_{3}\left(x, t_{3}\right)=0$ \\
\hline
\end{tabular}

also be calculated as $Q_{\text {up }}=\left(T_{h}-T_{0}\right) /\left(R_{\text {in-up }}+R_{\text {up }}\right)$ and $Q_{\text {down }}$ $=\left(T_{h}-T_{0}\right) /\left(R_{\text {in-down }}+R_{\text {down }}\right)$, respectively. The modeling results of $R$ for the GaAs- and Si-based test devices have been plotted in Fig. 3 together with the experimental data. Because the thermal conductivities of PECVD silicon dioxide and nitride layers are process dependent and vary with temperature, different values of $k_{1,2}$ are used to fit the data as indicated. All values of $k_{1,2}$ used are in the reasonable range as reported in the literature. ${ }^{18}$ The agreement between the analytical modeling results and the experimental data is fairly reasonable.

\section{B. Second generic analytical model}

The second analytical model is based on the separation of variables solution of Laplace's equation for multilayered structures. ${ }^{7}$ The top dielectric layer here needs to be truncated to the width of the top heat spreader (i.e., the width of the top dielectric layer is $w_{4}$ instead of $w_{3}$ ), so that the last matching of temperature and heat flux between two neighboring layers could be intentionally applied at the interface with the heat source. The truncating of the top dielectric layer has negligible effect since the ratio of the heater width and the top dielectric layer thickness is normally greater than 100. The dimensionless temperature $\theta_{i}(i=1,2,3,4)$ for different structural layers is defined here as $\theta_{i}=\left(T_{i}-T_{0}\right) / T_{0}$. In each layer, the positive $y$-direction is defined along the direction away from the heat source with the origin of $y$ at the layer surface closer to the heat source. The boundary conditions for different mounting configurations are listed in Table I, and all the other surfaces exposed to the ambient are treated as adiabatic. The separation of variables solutions of Laplace's equation from Eq. (1) is modified for the different layers as

$$
\begin{aligned}
\theta_{i}(x, y)= & \beta_{i, 0}\left(1-\gamma_{i, 0} y\right)+\sum_{n=1}^{\infty} \beta_{i, n}\left[\cosh \left(\alpha_{i, n} y\right)\right. \\
& \left.-\gamma_{i, n} \sinh \left(\alpha_{i, n} y\right)\right] \cos \left(\alpha_{i, n} x\right),
\end{aligned}
$$

where $\beta_{i, n}$ and $\gamma_{i, n}(i=1,2,3,4)$ are undetermined separation coefficients, and $\alpha_{i, n}=2 n \pi / w_{3}$ for $i=1,3$ and $\alpha_{i, n}=2 n \pi / w_{4}$ for $i=2,4$ in order to satisfy the requirement of no heat escape from all the lateral faces. The total thermal resistance $R$ for the heat spreader device can be evaluated as 
TABLE II. Separation coefficients $\gamma_{i, n}(i=3,4)$ calculated from the boundary conditions listed in Table I.

\begin{tabular}{lcccc}
\hline \hline & $\gamma_{3,0}$ & $\gamma_{3, n}, n=1,2,3 \ldots$ & $\gamma_{4,0}$ & $\gamma_{4, n}, n=1,2,3 \ldots$ \\
\hline Junction-side-up & $1 / d_{3}$ & $\operatorname{coth}\left(\alpha_{3, n} d_{3}\right)$ & 0 & $\tanh \left(\alpha_{4, n} d_{4}\right)$ \\
Junction-side-down & 0 & $\tanh \left(\alpha_{3, n} d_{3}\right)$ & $1 / d_{4}$ & $\operatorname{coth}\left(\alpha_{4, n} d_{4}\right)$ \\
Sandwich structure & $1 / d_{3}$ & $\operatorname{coth}\left(\alpha_{3, n} d_{3}\right)$ & $1 / d_{4}$ & $\operatorname{coth}\left(\alpha_{4, n} d_{4}\right)$ \\
\hline \hline
\end{tabular}

$$
\begin{aligned}
R= & w_{h}^{-1} \int_{-w_{h} / 2}^{w_{h} / 2} \frac{T_{0} \theta_{1}(x, 0)}{Q} d x=\frac{T_{0} \beta_{1,0}}{Q} \\
& +\frac{2 T_{0}}{Q w_{h}} \sum_{n=1}^{\infty} \frac{\beta_{1, n}}{\alpha_{1, n}} \sin \left(\frac{1}{2} \alpha_{1, n} w_{h}\right) .
\end{aligned}
$$

The boundary conditions listed in Table I can be applied directly to Eq. (8) to calculate separation coefficients $\gamma_{i, n}$ for the layers 3 and 4, as listed in Table II. At a heat source-free interface between any two layers, the continuity of temperature and normal heat flow relates $\gamma_{i, n}$ in the neighboring layers as follows:

$$
\begin{aligned}
\gamma_{i, 0} & =\frac{\left(k_{i+2} / k_{i}\right) \gamma_{i+2,0}}{1+\left(k_{i+2} / k_{i}\right) d_{i} \gamma_{i+2,0}}, \\
\gamma_{i, n} & =\frac{\tanh \left(\alpha_{i, n} d_{i}\right)+\left(k_{i+2} / k_{i}\right) \gamma_{i+2, n}}{1+\left(k_{i+2} / k_{i}\right) \tanh \left(\alpha_{i, n} d_{i}\right) \gamma_{i+2, n}} .
\end{aligned}
$$

Applying Table II to Eq. (10) yields $\gamma_{1, n}$ and $\gamma_{2, n}$ for the two neighboring dielectric layers below and above the heat source. With knowing $\gamma_{1, n}$ and $\gamma_{2, n}, \beta_{1, n}$ can be determined by coupling the interfacial conditions of the two neighboring dielectric layers. The heat flow from either side of the heat source stripe is an as-yet-unknown complicated function of $x$; however, the combined heat flow per unit area from both sides is by assumption the constant of $Q /\left(w_{h} l_{3}\right)$ at each point of $x$. Therefore we have

$$
-k_{1} \frac{\partial \theta_{1}(x, 0)}{\partial y}= \begin{cases}\frac{Q}{l_{3} w_{h}}+k_{2} \frac{\partial \theta_{2}(x, 0)}{\partial y}, & 0 \leq|x|<\frac{1}{2} w_{h} \\ k_{2} \frac{\partial \theta_{2}(x, 0)}{\partial y}, & \frac{1}{2} w_{h}<|x|<\frac{1}{2} w_{4} \\ 0, & \frac{1}{2} w_{4}<|x| \leq \frac{1}{2} w_{3} .\end{cases}
$$

The continuity of temperature at the interface between the two dielectric layers yields an additional expression as

$$
\theta_{1}(x, 0)=\theta_{2}(x, 0), 0 \leq|x| \leq \frac{1}{2} w_{4} .
$$

After substituting Eq. (8) into Eqs. (11) and (12), both sides of Eq. (11) are multiplied with $\cos \left(2 m \pi x / w_{3}\right) \quad(m$ $=0,1,2 \ldots)$ and integrated from 0 to $w_{3} / 2$ and both sides of Eq. (12) are multiplied with $\cos \left(2 m \pi x / w_{4}\right)(m=0,1,2 \ldots)$ and integrated from 0 to $w_{4} / 2$. The unknown separation constants $\beta_{1, n}$ cannot be determined directly from these operations. However, a set of linear equations with unknown coefficients $\beta_{i, n}(i=1,2)$ can be determined as

$$
\begin{aligned}
& k_{1} \gamma_{1,0} w_{3} \beta_{1,0}+k_{2} \gamma_{2,0} w_{4} \beta_{2,0}=J w_{h}, \\
& w_{4} \beta_{1,0}+\sum_{n=1}^{\infty} \frac{w_{3}}{n \pi} \sin \left(\frac{n \pi w_{4}}{w_{3}}\right) \beta_{1, n}-w_{4} \beta_{2,0}=0 \\
& m \pi k_{1} \gamma_{1, m} \beta_{1, m}+\frac{k_{2} \gamma_{2,0} w_{3}}{m \pi} \sin \left(\frac{m \pi w_{4}}{w_{3}}\right) \beta_{2,0} \\
& +\sum_{n=1}^{\infty} n k_{2} \gamma_{2, n} w_{3}\left\{\frac{\sin \left[\left(n \pi w_{3}+m \pi w_{4}\right) / w_{3}\right]}{n w_{3}+m w_{4}}\right. \\
& \left.+\frac{\sin \left[\left(n \pi w_{3}-m \pi w_{4}\right) / w_{3}\right]}{n w_{3}-m w_{4}}\right\} \beta_{2, n}=\frac{J w_{3}}{m \pi} \sin \frac{m \pi w_{h}}{w_{3}} \\
& \sum_{n=1}^{\infty} w_{3}\left\{\frac{\sin \left[\left(m \pi w_{3}+n \pi w_{4}\right) / w_{3}\right]}{n \pi w_{4}+m \pi w_{3}}\right. \\
& \left.+\frac{\sin \left[\left(n \pi w_{4}-m \pi w_{3}\right) / w_{3}\right]}{n \pi w_{4}-m \pi w_{3}}\right\} \beta_{1, n}-\beta_{2, m}=0 .
\end{aligned}
$$

Equation (13) is solved by computer program, and the final values of $\beta_{1, n}$ are substituted into Eq. (9) to yield $R$ for different mounting configurations.

\section{DICUSSION}

\section{A. Junction-side-up configuration}

A laser mounted in a junction-side-up configuration is more accessible to optical fibers and more isolated from bonding contaminants. In this configuration, the low thermal conductivity laser substrate and the active device layers become a dominant part of the thermal resistance. For the values listed in Fig. 1, Eq. (9) yields for the nominal thermal resistance $R$ as $R$ (nominal) $=17.74 \mathrm{~K} / \mathrm{W}$. To develop some feeling for the thermally important quantities, we show in Fig. 5(a) the results of factor of 2 variations in several dimensions about their nominal values. In order of importance (slope), the length of the laser test structure comes first. Since thermal resistance $R$ is defined as $\Delta T /$ (total power), it follows that $R$ scales as $1 / l_{3}$, and therefore halving the laser device length $l_{3}$ doubles $R$. Next it is seen that halving the heater stripe width $w_{h}$ to $50 \mu \mathrm{m}$ raises $R$ from 17.74 to $24.20 \mathrm{~K} / \mathrm{W}$, while doubling the width to $200 \mu \mathrm{m}$ reduces $R$ to $13.50 \mathrm{~K} / \mathrm{W}$. A factor of \pm 2 variations for the thickness of the dielectric layers $d_{1,2}$ accounts for +6.67 to $-3.41 \mathrm{~K} / \mathrm{W}$, while for the width of the copper heat spreader $w_{4}$, it accounts for -1.94 to $+4.32 \mathrm{~K} / \mathrm{W}$. Thinning of the GaAs substrate by a factor of 2 reduces $R$ by $3.86 \mathrm{~K} / \mathrm{W}$. The changes of $R$ are not so significant for factor of \pm 2 variations in the thickness of copper heat spreader $d_{4}$ and width of the GaAs substrate $w_{3}$. The results of factor of 2 variations in thermal conductivity values for different layers are also plotted in Fig. 5(a). We can see clearly that the dominant thermal resistance for junction-side-up mounted sample comes from the low thermal conductivity substrate and dielectric layers, as evidenced by the steep slopes of the curves. By increasing the thermal conductivities of the substrate and dielectric layers by a factor of $2, R$ decreases both about $20 \%-25 \%$. 
(a)

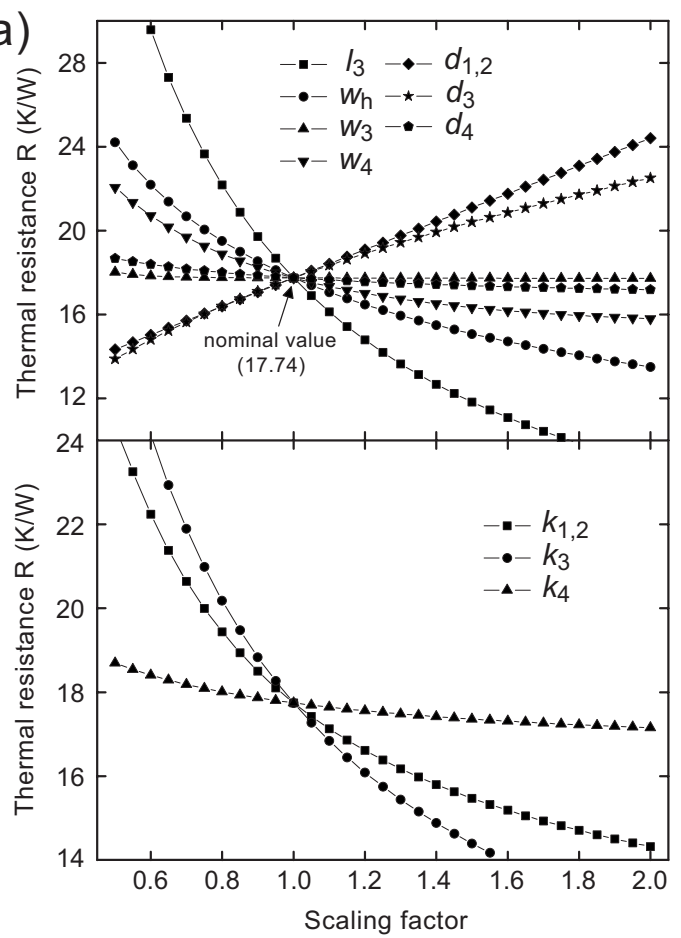

(b)

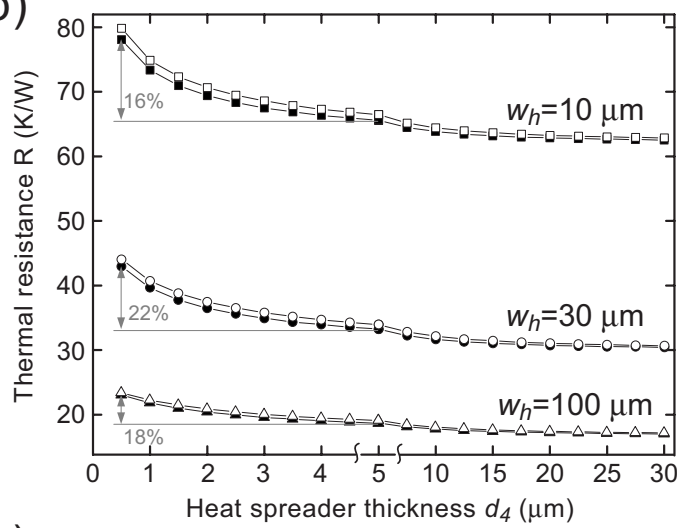

(c)

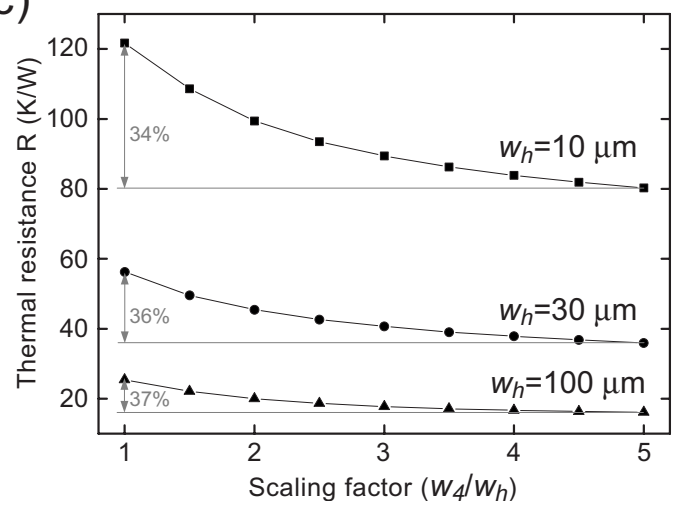

FIG. 5. Thermal resistance $R$ of the nominal laser test device in Fig. 1 mounted junction-side-up. (a) Thermal resistance $R$ as a function of factor of 2 changes in characteristic dimensions (top) and thermal conductivities (bottom). (b) Thermal resistance $R$ as a function of the heat spreader thickness $d_{4}$. Two metallic heat spreaders are included: $\mathrm{Cu}$ (solid symbols and $K_{4}$ $=398 \mathrm{~W} / \mathrm{m} \mathrm{K}$ ) and Au (open symbols and $K_{4}=317 \mathrm{~W} / \mathrm{m} \mathrm{K}$ ). (c) Thermal resistance $R$ as a function of the scaling factor of $w_{4} / w_{h}$.

Figure 5(b) shows detailed dependence of $R$ on the heat spreader thickness $d_{4}$ with two different metallic materials serving as the heat spreader. Clearly the heat spreader strongly reduces $R$, and most of the improvement is achieved with the first few microns of the heat spreader. Because of its higher thermal conductivity, the copper heat spreader achieves more reduction of $R$ than the gold heat spreader, although the difference is not very significant. Different test devices with different heating region widths are included in Fig. 5(b). Compared with a $0.5 \mu \mathrm{m}$ thick copper heat spreader, a $5 \mu \mathrm{m}$ thick copper heat spreader reduce $R$ of all the three laser devices (with heating region width $w_{h}$ of 10 , 50 , and $100 \mu \mathrm{m}$, respectively) by about $16 \%, 22 \%$, and $18 \%$, respectively. Figure 5(c) plots $R$ as a function of the scaling factor of heat spreader width (defined as $w_{4} / w_{h}$ ). With a scaling factor of 5, the thermal resistances of all the three devices (with heating region width $w_{h}$ of 10,50 , and $100 \mu \mathrm{m}$, respectively) are reduced by about $35 \%$. There is no significant difference of the heat spreading effect of the metallic heat spreader for laser devices with different heating region widths.

\section{B. Junction-side-down configuration}

For lasers mounted junction side down, the low thermal conductivity top device layers (e.g., layer 2) become a dominant part of the thermal resistance. The nominal thermal resistance $R$ is calculated as $R($ nominal $)=7.71 \mathrm{~K} / \mathrm{W}$, which is about $56 \%$ less than the nominal value for the lasers mounted junction side up. Figure 6(a) shows the results of factor of 2 variations in several dimensions about their nominal values. Similar to the lasers mounted junction side up, halving the laser structure length $l_{3}$ doubles $R$. Halving the heater stripe width $w_{h}$ to $50 \mu \mathrm{m}$ raises $R$ from 7.71 to 13.59 $\mathrm{K} / \mathrm{W}$, while doubling the width to $200 \mu \mathrm{m}$ reduces $R$ to 4.47 $\mathrm{K} / \mathrm{W}$. A factor of \pm 2 variations for the thickness of the dielectric layers $d_{1,2}$ accounts for +6.74 to $-3.52 \mathrm{~K} / \mathrm{W}$, while for the width of the copper heat spreader $w_{4}$, it accounts for -0.28 to $+1.02 \mathrm{~K} / \mathrm{W}$.

Factors of \pm 2 variations in any other dimensions (e.g., $w_{3}, d_{3}$, and $\left.d_{4}\right)$ account for less than about $\pm 0.14 \mathrm{~K} / \mathrm{W}$. In particular, halving the GaAs substrate width $w_{3}$ to $500 \mu \mathrm{m}$ raises $R$ by only $0.01 \mathrm{~K} / \mathrm{W}$, while halving the GaAs substrate thickness $d_{3}$ to $75 \mu \mathrm{m}$ raises $R$ by only $0.06 \mathrm{~K} / \mathrm{W}$. Therefore, from a thermal standpoint of view, the nominal laser structure is infinitely wide $\left(w_{3}=\infty\right)$ with an infinitely thick substrate $\left(d_{3}=\infty\right)$. Any heat sink additionally attached to the bottom of the substrate is of little value, since most of the heat generated in the heating region flows through the heat spreader to the top attached heat sink (this point will be further discussed in the next section). Figure 6(a) also plots the results of factor of 2 variations in thermal conductivity values for different layers. We can see clearly that the dominant thermal resistance for junction-side-down mounted sample comes from the low thermal conductivity top dielectric layer. By increasing the thermal conductivities of the layer 1 and 2 by a factor of $2, R$ decreases about $45 \%$.

For lasers mounted junction side down, the thickness of the copper heat spreader $d_{4}$ is roughly speaking, irrelevant and precisely speaking, somewhat counterproductive thermally in our devices. Figure 6(a) shows that doubling the heat spreader thickness $d_{4}$ to $20 \mu \mathrm{m}$ increases $R$ by $1.8 \%$ to $7.85 \mathrm{~K} / \mathrm{W}$. Only if there were voids or other interface imper- 
(a)
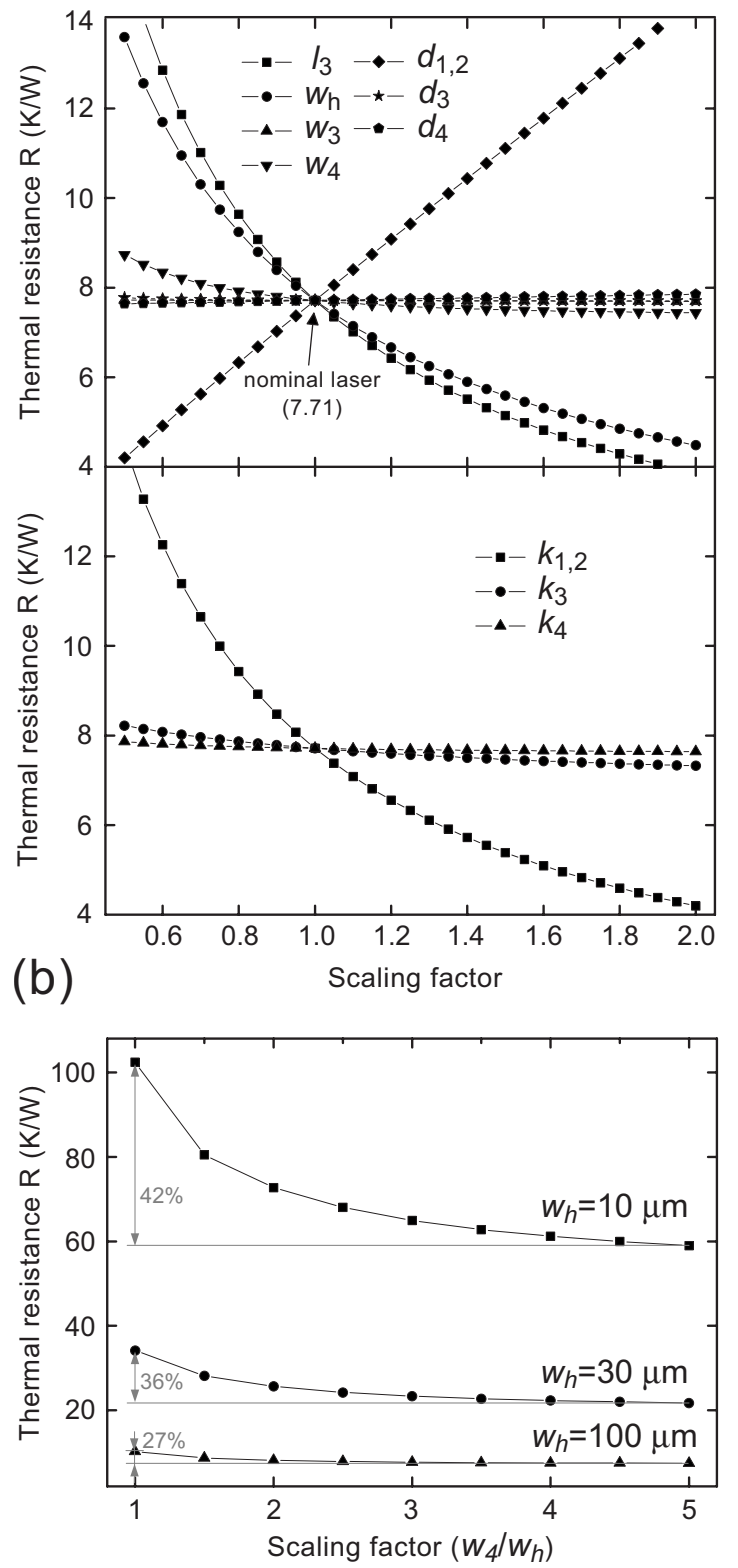

FIG. 6. Thermal resistance $R$ of the nominal laser test device in Fig. 1 mounted junction-side-down. (a) Thermal resistance $R$ as a function of factor of 2 changes in characteristic dimensions (top) and thermal conductivities (bottom). (b) Thermal resistance $R$ as a function of the scaling factor of $w_{4} / w_{h}$.

fections included in the soldering layer between the laser and the top heat sink (not included in Fig. 1) can a copper heat spreader on a good heat sink possibly reduce the thermal resistance $R$. Figure 6(b) shows detailed dependence of $R$ on the heat spreader width $w_{4}$. With a scaling factor of 5 , the thermal resistance $R$ of all three laser devices (with heating region width $w_{h}$ of 10,50 , and $100 \mu \mathrm{m}$, respectively) are reduced by about $42 \%, 36 \%$, and $27 \%$, respectively. The heat spreading effect of the copper heat spreader is more prominent for laser devices with narrower heating region widths, which is different from the junction-side-up configuration.

\section{Sandwich configuration}

The thermal characters of lasers mounted in a sandwich configuration are similar to the ones of lasers mounted junc- (a)
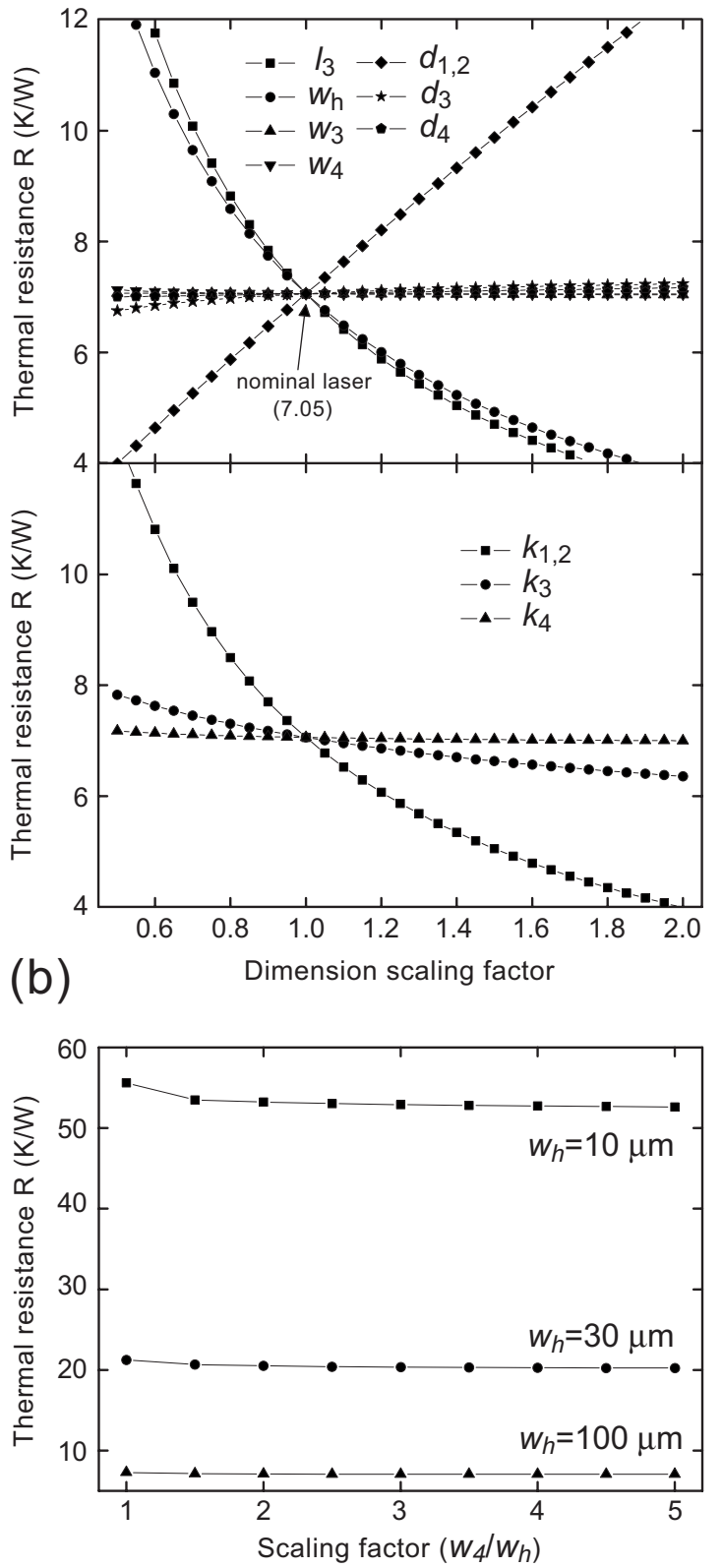

FIG. 7. Thermal resistance $R$ of the nominal laser test device in Fig. 1 in a sandwich configuration. (a) Thermal resistance $R$ as a function of factor of 2 changes in characteristic dimensions (top) and thermal conductivities (bottom). (b) Thermal resistance $R$ as a function of the scaling factor of $w_{4} / w_{h}$.

tion side down. The nominal thermal resistance $R$ is $R($ nominal $)=7.05 \mathrm{~K} / \mathrm{W}$, which is about $8 \%$ less than the nominal value for the lasers mounted junction side down. Figure 7(a) shows the results of factor of 2 variations in several dimensions about their nominal values. Halving the laser structure length $l_{3}$ doubles $R$. Halving the heater stripe width $w_{h}$ to $50 \mu \mathrm{m}$ raises $R$ from 7.05 to $12.94 \mathrm{~K} / \mathrm{W}$. A factor of \pm 2 variations for $d_{1,2}$ accounts for +5.51 to $-3.06 \mathrm{~K} / \mathrm{W}$. By increasing the thermal conductivities of the dielectric layers and the substrate by a factor of $2, R$ decreases about $43 \%$ and $10 \%$, respectively. Factors of \pm 2 variations in any other dimensions or conductivities account for less than about $\pm 0.3 \mathrm{~K} / \mathrm{W}$, and the thickness of the copper heat spreader $d_{4}$ is largely irrelevant thermally in our 
devices. Figure 7(b) plots $R$ as a function of the heat spreader width $w_{4}$. With a scaling factor of 5 , the thermal resistances of all the three devices (with heating region width $w_{h}$ of 10 , 50 , and $100 \mu \mathrm{m}$, respectively) are reduced by about $5.4 \%$, $4.8 \%$, and $3.4 \%$, respectively. The heat spreading effect of the copper heat spreader is more prominent for laser devices with narrower heating region widths, which is similar to the junction-side-down configuration, even though the difference is much smaller.

\section{SUMMARY AND CONCLUSIONS}

The objective of this work is to theoretically investigate heat spreading effect of the integrated electroplated metallic heat spreaders on high power semiconductor lasers under different mounting configurations. Using semiconductor microfabrication techniques, we have designed and tested a set of microdevices that mimic the thermal characteristics of the edge emitting semiconductor lasers. A microfabrication process has been developed that utilizes a thick negative photoresist NR9-8000 to generate thick photoresist molds for copper electroplating. Copper heat spreaders of different dimensions have been directly plated on top of the laser test structures to investigate their different heat spreading effect. Through experimental measurements, we have confirmed that the thermal resistances of the laser devices are strongly reduced by the top plated copper heat spreaders. Most of the benefit comes from the first few scaling factors. With a heat spreader scaling factor of 5 , the thermal resistances of both GaAs and Si laser devices reduce about $50 \%$ with junctionside-up mounting configuration.

In addition, we have developed two independent analytical models that can be utilized to calculate the thermal resistance of the heat spreader devices under different mounting configurations, without resolving to computational costive simulations. The first analytical model was developed to accommodate the design of the irregular meander-shaped heater in the laser device and therefore to explain the experimental data. Because of some of the assumptions made in the model, it is only suitable to calculate the thermal resistance of laser devices mounted junction side up. To further identify the dominant thermal resistance of the laser devices under other mounting configurations (e.g., junction-side-down and sandwich configuration), we further develop the second analytical model using the exact solution of a model in which two-dimensional heat flow is assumed to proceed from a uniformly excited heat strip to heat sinks of constant temperatures attached to either the bottom of the substrate or the top of the heat spreader (or both). Through comparison, we find our first analytical model that utilized a simplified thermal circuit agree fairly well with the experimental data. The thermal conductivity of the dielectric layers was used as a fitting parameter and the applied thermal conductivity values were in a reasonable range as compared to the values reported in the literature. The second two-dimensional analytical model has been utilized to investigate the influence of all the parameters affecting the thermal resistance of the laser devices under different mounting configurations. The dominant thermal resistance for junction-side-up mounted laser device has been found to stem from the low thermal conductivity substrate and dielectric layers. Most of the thermal resistance reduction by the top plated heat spreader is achieved within the first few microns of the heat spreader. No significant difference of the heat spreading effect has been found between laser devices with different heating region widths. The dominant thermal resistance for the junction-side-down mounted laser device was found from the low thermal conductivity top dielectric layer. For lasers mounted junction-side-down, the thickness of the copper heat spreader was found largely thermally irrelevant. Under junction-side-down configuration, the heat spreading effect of the copper heat spreader is more prominent for laser devices with narrower heating region widths, which is very different from the junction-side-up configuration. The thermal characters of lasers mounted in a sandwich configuration are similar to the ones mounted junction side down.

In summary, here we have demonstrated the use of a microfabricated laser test device to study the thermal management of the edge emitting semiconductor lasers. The approach of using such microfabricated devices to mimic semiconductors lasers allows us to study different aspects of the geometrical effects of the heat spreader structures on reducing the thermal resistance of high power semiconductor lasers. More importantly, the two analytical models developed in this work can be utilized to calculate the effects of different geometrical factors of the heat spreader on thermal resistance of lasers without resolving to computational costive simulations. Therefore, we believe our approach here of using microfabricated devices to mimic thermal characteristics of lasers as well as the developed analytical models for calculating the laser thermal resistance under different mounting configurations can potentially become valuable tools for thermal management of high power semiconductor lasers.

\section{ACKNOWLEDGMENTS}

We acknowledge financial support from DARPA HERETIC project (through JPL). We acknowledge valuable comments and suggestions on the manuscript by S. Pei and C. Dames. The UCLA Microfabrication Laboratory is acknowledged for support in fabrication.

\footnotetext{
${ }^{1}$ D. F. Welch, IEEE J. Sel. Top. Quantum Electron. 6, 1470 (2000).

${ }^{2}$ High-Power Diode Lasers, Fundamentals, Technology, Applications, edited by R. Diehl (Springer, New York, 2000).

${ }^{3}$ G. Hasnain, K. Tai, L. Yang, Y. H. Wang, R. J. Fischer, J. D. Wynn, B. Weir, N. K. Dutta, and A. Y. Cho, IEEE J. Quantum Electron. 27, 1377 (1991).

${ }^{4}$ J. W. Scott, R. S. Geels, S. W. Corzine, and L. A. Coldren, IEEE J. Quantum Electron. 29, 1295 (1993).

${ }^{5}$ Y. Ueno, K. Endo, H. Fujii, K. Kobayashi, K. Hara, and T. Yuasa, Electron. Lett. 26, 1726 (1990).

${ }^{6}$ G. Chen, Annu. Rev. Heat Transfer 7, 1 (1996).

${ }^{7}$ W. B. Joyce and R. W. Dixon, J. Appl. Phys. 46, 855 (1975).

${ }^{8}$ T. Kobayashi and G. Iwane, Jpn. J. Appl. Phys. 16, 1403 (1977).

${ }^{9}$ D. H. Newman, D. J. Bond, and J. Stefani, IEE J. Solid-State Electron Devices 2, 41 (1978).

${ }^{10}$ S. M. S. Kastigar, R. E. Hendron, J. R. Lapinski, and G. R. Hertzler, Proc. SPIE 1043, 359 (1989).

${ }^{11}$ W. Nakwaski, J. Appl. Phys. 67, 2711 (1990).

${ }^{12}$ Y. Lin and Z. Fang, Electron. Lett. 27, 18 (1991).

${ }^{13}$ O. J. F. Martin, G.-L. Bona, and P. Wolf, IEEE J. Quantum Electron. 28, 2582 (1992).
} 
${ }^{14}$ B. Beach, W. J. Benett, B. L. Freitas, D. Mundinger, B. J. Comaskey, R. W. Solarz, and M. A. Emanuel, IEEE J. Quantum Electron. 28, 966 (1992).

${ }^{15}$ A. Yariv, Quantum Electronics, 3rd ed. (Wiley, New York, 1989).

${ }^{16}$ J. Faist, F. Capasso, D. L. Sivco, C. Sirtori, A. L. Hutchinson, and A. Y. Cho, Science 264, 553 (1994).

${ }^{17}$ G. Chen, Semicond. Semimetals 71, 203 (2001).
${ }^{18}$ S.-M. Lee and D. G. Cahill, J. Appl. Phys. 81, 2590 (1997).

${ }^{19}$ W. W. Flack, S. White, and B. Todd, Proceedings of the SPIE Microlithography, 1999 (unpublished), Paper No. 3678-49.

${ }^{20}$ A. B. Frazier, Ph.D. thesis, Georgia Institute of Technology, 1993.

${ }^{21}$ M. T. Wang, M. S. Tsai, and C. Liu, Thin Solid Films 308-309, 518 (1997).

${ }^{22}$ M. N. Ozisik, Heat Conduction, 2nd ed. (Wiley, New York, 1993). 